\title{
Correction to: Erythropoietin Abrogates Post-ischemic Activation of the NLRP3, NLRC4, and AIM2 Inflammasomes in Microglia/ Macrophages in a TAK1-Dependent Manner
}

\author{
Ole Heinisch ${ }^{1} \cdot$ Thomas Zeyen $^{1,2} \cdot$ Tobias Goldmann $^{3} \cdot$ Marco Prinz $^{3,4,5} \cdot$ Michael Huber $^{6}$. Jennifer Jung ${ }^{1}$. Eren Arik ${ }^{1}$. \\ Shahin Habib ${ }^{7}$. Alexander Slowik ${ }^{8}$ - Arno Reich ${ }^{1}$. Jörg B. Schulz ${ }^{1,9} \cdot$ Pardes Habib $^{1,6,9}$
}

Published online: 6 November 2021

๑) Springer Science+Business Media, LLC, part of Springer Nature 2021

\section{Correction to: Translational Stroke Research https://doi.org/10.1007/s12975-021-00948-8}

Unfortunately, there were some mistakes on the original published paper.

1. Figures 1, 2 and 3 were incorrectly positioned in the PDF and appear under the material and methods section.

2. The correct email address of Thomas Zeyen is: thomas. zeyen@ukbonn.de.

The original article can be found online at https://doi.org/10.1007/ s12975-021-00948-8.

Pardes Habib

phabib@ukaachen.de

Ole Heinisch

ole.heinisch@rwth-aachen.de

Thomas Zeyen

thomas.zeyen@ukbonn.de

Tobias Goldmann

tobias.goldmann@gmail.com

Marco Prinz

marco.prinz@uniklinik-freiburg.de

Michael Huber

mhuber@ukaachen.de

Jennifer Jung

jennifer.jung@rwth-aachen.de

Eren Arik

eren.arik@rwth-aachen.de

Shahin Habib

sh720@student.le.ac.uk

Alexander Slowik

aslowik@ukaachen.de
The original article has been corrected.

Publisher's Note Springer Nature remains neutral with regard to jurisdictional claims in published maps and institutional affiliations.
Arno Reich

areich@ukaachen.de

Jörg B. Schulz

jschulz@ukaachen.de

1 Department of Neurology, Medical Faculty, RWTH Aachen University, Pauwelsstraße 30, D-52074 Aachen, Germany

2 Department of Neurology, University Hospital of Bonn, Bonn, Germany

3 Institute of Neuropathology, Faculty of Medicine, University of Freiburg, Freiburg, Germany

4 Signalling Research Centres BIOSS and CIBSS, University of Freiburg, Freiburg, Germany

5 Center for Basics in NeuroModulation (NeuroModulBasics), Faculty of Medicine, University of Freiburg, Freiburg, Germany

6 Institute of Biochemistry and Molecular Immunology, Medical Faculty, RWTH Aachen University, Aachen, Germany

7 Medical Biochemistry, Department of Biochemistry, University of Leicester, Leicester, UK

8 Department of Anatomy and Cell Biology, Medical Faculty, RWTH Aachen University, Aachen, Germany

9 JARA-BRAIN Institute of Molecular Neuroscience and Neuroimaging, Forschungszentrum Jülich GmbH, RWTH Aachen University, Aachen, Germany 